\title{
Directionality Edge Detection Algorithm Based on Direction Wavelet Transform
}

\author{
Shi Tong YE* \\ Huali College Guangdong University of Technology, Guangzhou Zengcheng 511325, China \\ yst888_0@126.com
}

Keywords: Edge detection algorithm; Wavelet decomposition; Directional characteristics; Search window.

\begin{abstract}
Existing edge detection algorithms have the problem of that poor directionality and low definition. In response to this phenomenon, this paper put forward a directionality edge detection algorithm using layered processing. This algorithm by analyzing wavelet decomposition directional characteristics of high frequency sub-bands. And introduction of a direction factor in traditional edge detection algorithm, which realize edge detection of multi-directional detection. In the process of edge detection, article algorithm reprocessing the weak edge signal so that it can better protection of the edge signal. This algorithm compared with the existing edge detection algorithm, this algorithm with the better performance of the fine image structure and object Edge.
\end{abstract}

\section{Introduction}

Edge detection algorithm is an important task in image processing, image analysis and recognition field. It is widely used in medical and military. Its essence is the extraction technology of the boundary line between the target object and the background. Therefore, how quickly and accurately extract the edge signal has always been a hot research in domestic and foreign. Early have many edge algorithms[1, 2]. For exmaple: Edge operator, Template matching method, Thresholding method,etc. In recent years, there are many new edge detection algorithm[3, 4, 5]. For exmaple: Based on wavelet transform, Mathematical Morphology, Neural Networks,etc. But through the study found that early edge extraction algorithm is relatively simple in design and computing speed, but these has some problem of weak noise immunity and low marginal accuracy. For the algorithm proposed in recent years, Although these algorithms can achieve more early desired effect than the edge extraction algorithm. But these algorithm still exists in many areas for improvement, such as Easy to produce false edge and edge sharpness lower.

Through the study of existing algorithms, we have found that the traditional wavelet edge detection algorithm lack of flexible using the directional characteristics of wavelet. So lead the poor continuity and low resolution in the edge image[6]. Therefore, this paper proposed a new edge detection algorithm that combination of the directional characteristic and edge extraction characteristics. Through the direction search, edge search and the reprocessing, we can get the higher accuracy of edge information. Experimental results show that it can effectively extracted edge signals. That was results in line with expectations. 


\section{Edge detection algorithm based on wavelet}

After wavelet transform[7], get this: $W_{\mathrm{f}}(a, b)=\frac{1}{\sqrt{C_{\psi}}} \frac{1}{\sqrt{|a|}} \int_{-\infty}^{+\infty} f(t) \bar{\psi}\left(\frac{t-b}{a}\right) d t$

Therein $C_{\psi}=2 \pi \int_{-\infty}^{+\infty} \frac{|\psi(\omega)|^{2}}{|\omega|} \mathrm{d} \omega, \psi(\omega)$ is Fourier transform with $\psi(t)$, a is stretch factor, b is shift factor; Hypothesis $\theta(\mathrm{x}, y)$ satisfy the following conditions: $\left\{\begin{array}{l}\iint_{R^{2}} \theta(\mathrm{x}, y) d x d y=1 \\ \lim _{x, y \rightarrow+\infty} \theta(x, y)=0\end{array}\right.$

$\theta(x, y)$ is smoothing function. it was satisfies with the wavelet function: $\left\{\begin{array}{l}\psi^{1}(x, y)=\frac{\partial \theta(x, y)}{\partial x} \\ \psi^{2}(x, y)=\frac{\partial \theta(x, y)}{\partial y}\end{array}\right.$

Therein $\psi^{1}(x, y) 、 \psi^{2}(x, y)$ are basis function of wavelet. 1.e.: $\left\{\begin{array}{l}\psi^{1}(x, y)=\frac{1}{2^{2}} \psi^{1}\left(\frac{\mathrm{x}}{2^{j}}, \frac{\mathrm{y}}{2^{j}}\right) \\ \psi^{2}(x, y)=\frac{1}{2^{2}} \psi^{2}\left(\frac{\mathrm{x}}{2^{j}}, \frac{\mathrm{y}}{2^{j}}\right)\end{array}\right.$

When the scale of $2^{j}$, Horizontal and vertical directions is: $\left\{\begin{array}{l}W_{2^{j}}^{1} f(x, y)=f * \psi_{2^{j}}^{1} f(x, y) \\ W_{2^{j}}^{2} f(x, y)=f * \psi_{2^{j}}^{2} f(x, y)\end{array}\right.$

Written as: $W_{2^{j}} f(x, y)=\left[\begin{array}{l}W^{1}{ }^{j} f(x, y) \\ W^{2}{ }^{j} f(x, y)\end{array}\right]=2^{j}\left[\begin{array}{c}\frac{\partial}{\partial x} f \theta_{2^{j}}(x, y) \\ \frac{\partial}{\partial y} f \theta_{2^{j}}(x, y)\end{array}\right]=2^{j} \nabla\left(f \theta_{2^{j}}\right)(x, y)$

L.e. modulus and argument expression function of the wavelet coefficients is

Mold: $M_{2^{\mathrm{j}}} f(x, y)=\sqrt{\left|W_{2^{\mathrm{j}}}^{1} f(x, y)\right|^{2}+\left|W_{2^{\mathrm{j}}}^{2} f(x, y)\right|^{2}}$ (7) Argument: $\left.A_{2^{j}} f(x, y)=\arctan \frac{W_{2^{j}}^{2} f(x, y)}{W_{2^{j}}^{1} f(x, y)}\right)$

\section{Article algorithm}

Wavelet analysis. The study found that the wavelet transform have characteristics of a certain direction. Through the wavelet decomposition, can get high frequency sub-signals in different directions. These are having certain rules at each high frequency sub-band. Wavelet decomposition results were shown below.

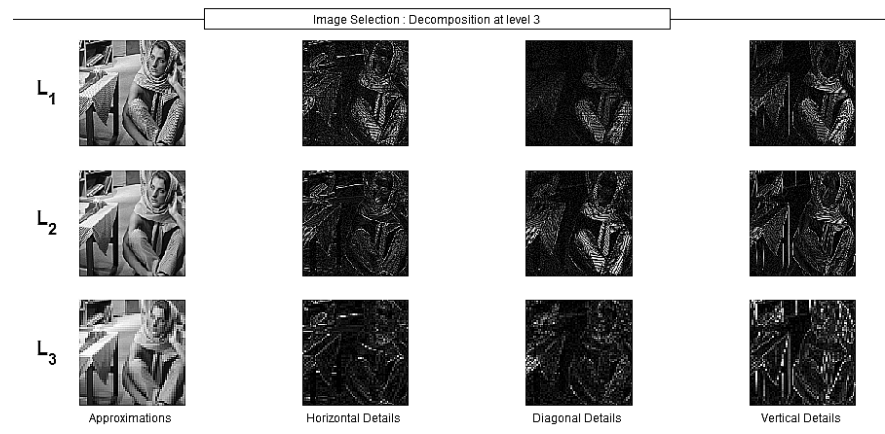

Figure. 1 Sub-image of wavelet decomposition

By observing Figure 1, we can see that high frequency detail signal having horizontal、vertical direction and the diagonal direction[8]. And in the process of decomposition, Details of the signal are layer by layer to weaken. 
Edge detection algorithm with the direction. By wavelet edge detection algorithm found that definition quality of the smoothing function will determine the accuracy. Therefore, we were re-definition for smoothing function. and according to the symmetric Gaussian function. smooth

function: $\theta_{a}(x, y)=\frac{1}{2 \delta^{2}} \exp \left(-\frac{x^{2}+y^{2}}{2 \delta^{2}}\right)$

This paper introducing an orientation factor $\theta$ Based on the original. Defined as follows.

$$
\begin{aligned}
& W_{a}^{\theta} f(x, y)=W_{a}^{x} f(x, y) \cos \theta+W_{a}^{y} f(x, y) \sin \theta=|| W_{a} f(x, y)||\left(\frac{W_{a}^{x} f(x, y)}{\left\|W_{a} f(x, y)\right\|} \cdot \cos \theta+\frac{W_{a}^{y} f(x, y)}{\left\|W_{a} f(x, y)\right\|} \cdot \sin \theta\right) \\
& =\mid W_{a} f(x, y) \|\left(\cos \left(\operatorname{Arg}\left[W_{a} f(x, y)\right]\right) \cdot \cos \theta+\sin \left(\operatorname{Arg}\left[W_{a} f(x, y)\right]\right) \cdot \sin \theta\right) \\
& =\left|W_{a} f(x, y)\right||(\cos \alpha \cos \theta+\sin \alpha \sin \theta)=|\left|W_{a} f(x, y)\right| \mid \cos (\alpha-\theta)
\end{aligned}
$$$$
W_{a}^{x} f(x, y)=\mathrm{f}(x, y) * \psi_{a}^{x}(x, y)=\mathrm{f}(x, y) * \frac{1}{2^{j}} \theta^{x}\left(\frac{\mathrm{x}}{2^{j}}, \frac{\mathrm{y}}{2^{j}}\right)=\frac{1}{2^{j}}\left(-\frac{1}{2 \delta^{4}}\right) f(x, y) * x \exp \left(-\frac{x^{2}+y^{2}}{2^{j} 2 \delta^{2}}\right)
$$

Hypothesis $k=-2^{j} 2 \delta^{2}$, so $W_{a}^{x} f(x, y)=\frac{1}{k \delta^{2}} f(x, y) * x \exp \left(\frac{x^{2}+y^{2}}{k}\right)$

Similarly available, $W_{a}^{y} f(x, y)=\frac{1}{k \delta^{2}} f(x, y) * y \exp \left(\frac{x^{2}+y^{2}}{k}\right)$

The high-frequency subbands in different directions to adjust the angle of the detection algorithm. Which process is as follows.

The basis functions $\psi(x, y)$ for wavelet, Take $\theta=0 、 \pi / 4 、 \pi / 2 、 3 \pi / 4$. In here, this paper was based on the direction of each sub-band high frequency characteristics. Make horizontal high-frequency sub-band select $\theta=0$, Vertical high-frequency sub-band select $\theta=\pi / 2$, and diagonal high-frequency sub-band select $\theta=\pi / 4 、 \pi / 4$.

Calculate that $W_{a}^{0} f(x, y) 、 W_{d}^{\frac{\pi}{4}} f(x, y) 、 W_{a}^{\frac{\pi}{2}} f(x, y)$ 和 $W_{a}^{\frac{3 \pi}{4}} f(x, y)$, and using the result to calculate the coefficient of the high frequency sub-bands in all directions.

Then get the edge map are horizontal, vertical and diagonal directions between the high frequency sub-bands. Fusion edge signal all directions can get the ideal edge.

When we definition the scale functions $\mathrm{a}=2^{j}$, Mode functions as follows.

Mode functions: $M_{2^{j}}^{\theta} f(x, y)=\sqrt{\left|W_{2^{\theta^{j}}}^{\theta^{1}} f(x, y)\right|^{2}+\left|W_{2^{j}}^{\theta^{2}} f(x, y)\right|^{2}}$

Determine the signal of the edge. In the judgment phase, this paper is judge the edge of the edge information at first [9]. When $M_{2^{j}}^{\theta} f(x, y) \geq \tau_{H}$, it indicates that the pixel is an edge point. When $M_{2^{j}}^{\theta} f(x, y) \leq \tau_{L}$, it indicates that the pixel is not edge point. When $\tau_{L}<M_{2^{j}}^{\theta} f(x, y)<\tau_{H}$, judged again with the edge single.

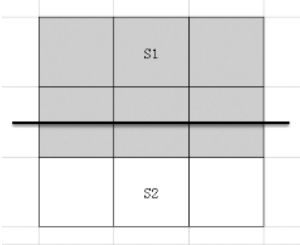

(a)edge direction 1(horizontal)

(c) edge direction 3(Diagonal 1)

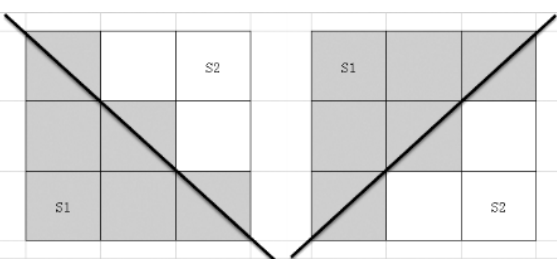

(b) edge direction 2(Vertical)

(d) edge direction 4(Diagonal 2)

Figure. 2 edge points of the search window $3 \times 3$ 
According to the characteristics of wavelet transformation, Selection grayscale L, and make the gradation values in the range of [0, L-1]. This paper was using the search window $3 \times 3$ to Search. Using the following three directions to instead eight kinds of edge directions. Method is as follows.

By using the above four kinds of search window, we can get the edge information of high-frequency subbands. Search programs to each frequency subband is as follows:

According to the edge of the gray values are divided into two groups, that was $S_{0} 、 S_{1}$. Calculating the average of its. L.e. $m_{0}=\frac{1}{6} \sum P_{i}, P_{i} \in S_{0} \quad ; \quad m_{1}=\frac{1}{3} \sum P_{i}, P_{i} \in S_{1}$

In order to more accurately predict the intensity and direction of the edge signal, we need to define the following two functions

$N_{\mathrm{k}}=\min \left(1, \frac{\left|m_{0}-m_{1}\right|}{W_{1}}\right) ; k=1,2,3 \quad ; \quad D_{k}=1+\frac{1}{15} \sum_{P_{m}, P_{n} \in S_{0}} \min \left(1, \frac{\left|P_{m}-P_{n}\right|}{W_{2}}\right)+\frac{1}{3} \sum_{P_{m}, P_{n} \in S_{0}} \min \left(1, \frac{\left|P_{m}-P_{n}\right|}{W_{2}}\right)$

therein $\mathrm{m}>n$ 且 $k=1,2,3, \quad W_{1}$ and $W_{2}$ is the threshold after the text.

Assuming that the target function $f_{k}$ for each direction, Function is expressed as follows:

$f_{k}=(L-1) \frac{N_{k}}{D_{k}}, k=1,2,3$ L.e. $W_{a}^{\theta} f_{k}(x, y)=|| W_{a} f_{k}(x, y)|| \cos (\alpha-\theta)$

Make horizontal high-frequency sub-band select $\theta=0$, Vertical high-frequency sub-band select $\theta=\pi / 2$, and diagonal high-frequency sub-band select $\theta=\pi / 4 、 \pi / 4$.

We can get this function $M_{2^{j}}^{\theta} f_{k}(x, y)$. When $M_{2^{j}}^{\theta} f_{k}(x, y)>\tau$, it indicates that the pixel is an edge

point. When $M_{2^{j}}^{\theta} f_{k}(x, y)>\tau$, it indicates that the pixel is not edge point.

\section{Simulation experiments}

Simulation experiments was using Matlab 7.10.0. And in order to detect the feasibility of the proposed algorithm, we were using article to compare with other algorithms.

Table. 1 Classification table of the image edge

\begin{tabular}{ccc}
\hline edge & characteristic & Image Name \\
\hline $0.4 \sim 0.6$ & textures / Edge & baboon、goldhill、 plant \\
$0.2 \sim 0.1$ & textures / Edge / Smooth & lena、pepper、boat \\
$0.1 \sim 0.2$ & Edge / Smooth & circle、pillsetc、gray \\
\hline
\end{tabular}

comparative experiments. Compare with the Sobel operator, traditional wavelet algorithm for edge detection algorithms and literature algorithm. And select the liftingbody and rose to text, which these are represent for simple and complex images.

Through observation, we can find this algorithm in edge sharpness and detail are better than other algorithms. The resulting simulation figure from Sobel operator is vague. And it has more virtual signals, beause these are disturbed by the background signal. Form using edge detection algorithm based on wavelet transform, we can find that it can be reducing interference, but the continuity of the image is poor. In comparison, the result of article algorithm was close to the literature [10] algorithm. But article algorithm can get a better edge single in the simulation figure. It can making weak edge to be better protected. 


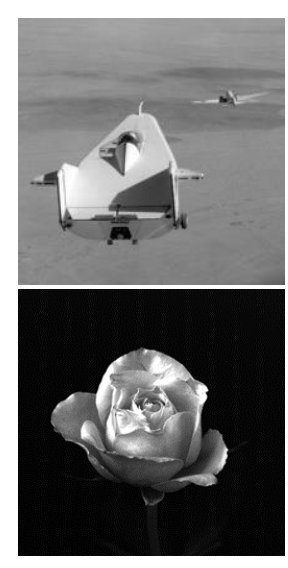

(a) Original image

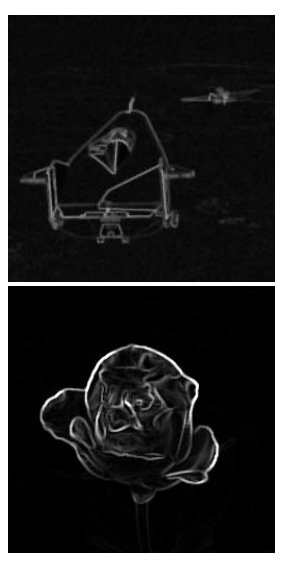

(b) Sobel operator algorithm[10]
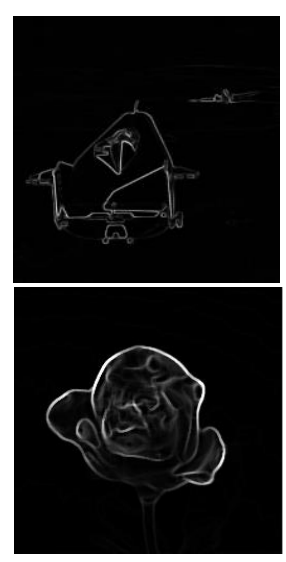

(c) Edge detection based on wavelet

(e) Article algorithm
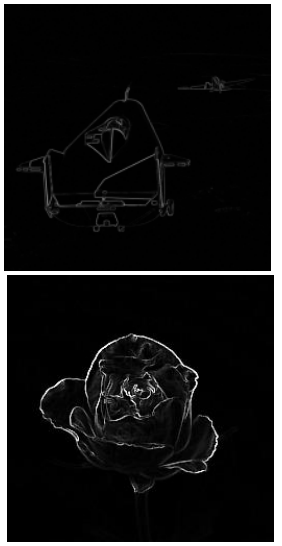
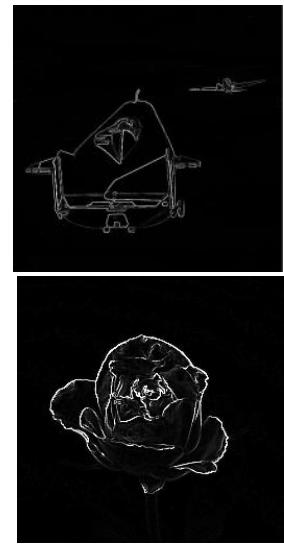

Figure. 3 simulation map of each algorithm

\section{Conclusion}

This paper proposed a direction of edge detection algorithm. It was according to the direction characteristics of wavelet transform. And it was using a targeted search window to increase the extraction capacity of wavelet coefficients in the high-frequency sub-bands. And it was combined with the direction of the edge detection algorithm to extracting the edge signal. Experimental results show that article algorithm can maintain a high accuracy in different image. Compared with the other edge of the algorithm, it has advantages of high definition and strong anti-interference.

\section{References}

[1] Lira J, Rodriguez A. Edge enhancement in multispectral satellite images by means of vector operators [J]. GEOFISICA INTERNACIONAL. 2014,53(3): 289-308.

[2] Hiremath PS, Tegnoor JR. Fuzzy inference system for follicle detection in ultrasound images of ovaries [J]. SOFT COMPUTING. 2014,18(7): 1353-1362.

[3] Chavez-Roman H, Ponomaryov V. Super Resolution Image Generation Using Wavelet Domain Interpolation With Edge Extraction via a Sparse Representation [J]. IEEE GEOSCIENCE AND REMOTE SENSING LETTERS. 2014, 11(10): 1777-1781.

[4] Zhen-yun D, Xiao-feng L, Xu Y. Edge extraction algorithm based on anisotropic diffusion filtering [J]. Journal of Shenyang University of Technology. 2014,36(4): 421-425.

[5] Wang XL, Zhang W, Ji Q. Image object extraction with shape and edge-driven Markov random field model [J]. 2014,8(7):383-396.

[6] Nes PG. Fast multi-scale edge-detection in medical ultrasound signals [J]. SIGNAL PROCESSING. 2012, 92(10): 2394-2408.

[7] Guo FD, Yang YH, Chen B, Guo LJ. A novel multi-scale edge detection technique based on wavelet analysis with application in multiphase flows [J]. POWDER TECHNOLOGY. 2010,202(1-3): 171-177.

[8] Xu HN, Peng GH, Liu Z. New Wavelet Based Directional Transform [J]. Computer Science. 2014,41(3): 282-285.

[9] Fan YJ, Wu XH, Luo DS. A modified image edge detection algorithm based on wavelet transform [J]. Sichuan Daxue Xuebao (Ziran Kexueban). 2012,49(6): 1264-1268.

[10]Zhang HL, Ravat D, Marangoni YR, Hu XY. NAV-Edge: Edge detection of potential-field sources using normalized anisotropy variance [J].GEOPHYSICS. 2014,79(3): 43-53. 